\title{
A Heat-Loss-Compensated Calorimeter: Theory, Design, and Performance*
}

\author{
Steve R. Domen and Paul J. Lamperti \\ Institute for Basic Standards, National Bureau of Standards, Washington, D.C. 20234
}

(April 17, 1974)

\begin{abstract}
A new type of 3-body calorimeter for measuring absorbed dose produced by ionizing radiation is described in detail. All three bodies rise in temperature during irradiation, and the heat absorbed by the central core is measured by standard means. Only the central core is heated during electrical calibration, but the increased heat losses are compensated by measuring most of the heat lost to the surrounding jacket and automatically adding it to the heat retained by the core. The third body is a massive, thermally-floating shield, whose presence reduces the heat losses during irradiation, with a consequent increase in sensitivity and stability. A mathematical description of the calorimeter behavior is presented, along with a discussion of control and operation technique. In particular, it is shown how this 3-body calorimeter can be calibrated as a 1-body calorimeter, with large heat losses, or as a 2 -body calorimeter, in the quasi-adiabatic mode. This calorimeter design decreases the effects of thermal gradients and at the same time provides the means to test for these effects. The results of these tests show that for this particular model, systematic errors caused by thermal gradients, during electrical measurements, are no larger than 0.1 percent. Errors in comparing an electrical run with an irradiation may be somewhat larger because of different temperature gradients within the system. It is also pointed out that the general design of this calorimeter is not restricted to measuring absorbed dose but can be applied to calorimetry in general.
\end{abstract}

Key words: Absorbed dose; calorimeter; heat-loss-compensation; thermal gradients.

\section{Introduction}

The purpose of this report is to describe a new type of calorimeter developed at the National Bureau of Standards for measuring absorbed dose ${ }^{1}$ delivered by ionizing radiation. The basic principles have already been described [1-3], ${ }^{2}$ but will be repeated here, along with a detailed description of the construction and use of the present models. It is believed that this general design could be used to advantage in conventional calorimetry, as well as in the more specialized absorbed-dose field, and the description immediately following is intended to be generally applicable.

Historically, one of the principal limitations on the accuracy of an isoperibolic calorimeter (a thermally insulated body in a constant-temperature environment) has been the accuracy with which corrections could be made for heat lost to the surroundings [4]. In the new design, most of this heat is compensated for by being measured and automatically added to the

* This work was supported primarily by the National Cancer Institute. National Institutes of Health, Bethesda, Maryland. Some initial work was supported by the Division of Biology and Medicine of the United States Atomic Energy Commission, Washington, D.C.

1 Absorbed dose is the quotient of the mean energy imparted by ionizing radiation to the mass in an element of volume. The special unit of absorbed dose is the rad. $1 \mathrm{rad}=$ the mass in
$10^{-2} \mathrm{Jkg}^{-1}$.

${ }^{2}$ Figures in brackets indicate literature references at the end of this paper. heat retained. This is done by enclosing the core of the calorimeter, where the heat is introduced, in a thermally insulated jacket, and electrically measuring and adding their temperature rises. If the two components have equal heat capacities, the sum of their temperature rises is proportional to the sum of the heat retained by each. There will still be a correction for heat escaping from the jacket, but its magnitude will be considerably smaller, because the jacket is not heated directly, and its temperature rise will be much smaller than that of the core. Moreover, the jacket temperature necessarily will be more uniform than that of the core. Hence a comparison of measurements made with and without heat-loss compensation should provide a measure of the effects of thermal gradients in the core. ${ }^{3}$

In absorbed dose work, the components near the core are heated uniformly when the calorimeter is irradiated with high-energy beams, so that core thermal gradients are negligible and the core loses very little heat. During electrical calibrations, however, when an accurately measured quantity of heat is dissipated in the core, its temperature will rise above that of the surroundings,

\footnotetext{
${ }^{3}$ Reference [5] describes a somewhat similar method of determining heat losses, but makes no mention of thermal gradients. Reference [6] describes an alternative method for reducing the effects of thermal gradients.
} 
and there may be significant heat loss. Historically, this situation has been avoided by the development of the quasi-adiabatic calorimeter [7] where equal electrical power densities are dissipated in core and jacket, but where only the core temperature rise is measured. Calibrations using this technique closely simulate the radiation case, except for thermal gradients in the core caused by localized dissipation of electrical power. Typically, these gradients have been reduced by constructing the core like a sandwich, in an attempt to dissipate the electrical power uniformly throughout the thin middle layer [7-9].

The design described in this report offers a valuable alternative to the quasi-adiabatic calorimeter for adsorbed-dose measurements. The heat-loss corrections for electrical calibrations and the effects of thermal gradients are small because of the dual role played by the jacket, which must be considered an integral part of the new calorimeter. Moreover, as already mentioned, comparison of corrected calorimeter calibrations with and without heat-loss compensation provides an excellent test of the effects of thermal gradients. In addition, the calorimeter can be operated in a quasiadiabatic mode, and a comparison of its calibration in the two modes serves as an additional consistency check.

This report includes a comprehensive mathematical description of the new calorimeter operation, and a discussion of the sources of systematic error in such an instrument. This is followed by detailed descriptions of the calorimeter construction and techniques of operation. The results of the bench tests discussed above are described, and a brief summary of radiation experiments is included. The final section is a discussion of some of the results and a suggestion as to how this calorimeter might be improved.

\section{Calorimeter Theory}

Figure 1 is a schematic diagram of the calorimeter. It consists of three components, core, jacket, and

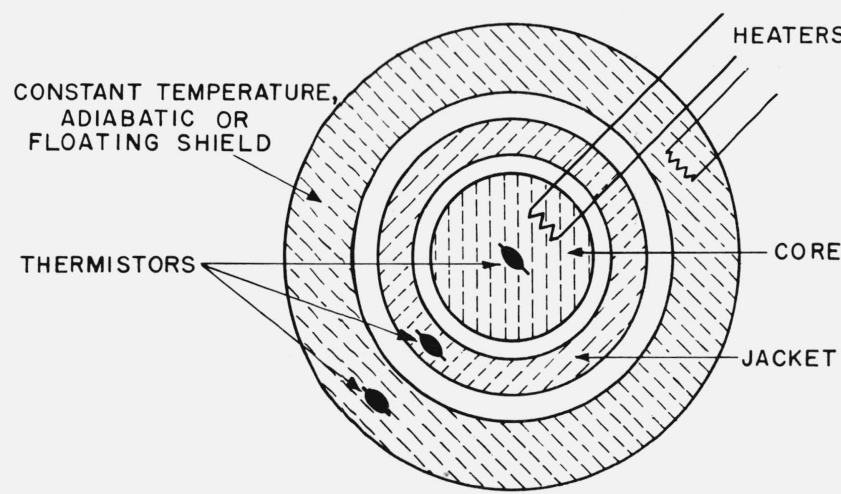

Figure 1. Essential elements of the heat-loss-compensated calorimeter. Thermistors of equal sensitivity are embedded in a core and in a jacket of equal heat capacity.

A thermistor and a heater are embedded in the shield to aid in restoring temperature equilibrium. Adiabatic or floating modes of the shield require a surrounding medium regulated at constant temperature. shield, all made of the same material. The core and jacket have equal heat capacities, while that of the shield is considerably larger. The three components contain thermistors of equal sensitivity, which are used for temperature measurements. In addition, the core and shield each contain an electrical heater. The core heater is used for calibration, and both heaters are used to restore the system to equilibrium after a run, as described in section 5 . The three bodies are thermally insulated from each other, and from a surrounding medium (not shown) which is made of the same material, and is maintained at a constant temperature.

In the original proposals for a heat-loss-compensated calorimeter [1-3], it was suggested that the shield temperature could be controlled, either to follow the jacket temperature adiabatically or to remain constant. That proposed calorimeter will be called the 2-body model, since only the temperature rise of the core and of the jacket are of interest. The calorimeter described in this report, which has a thermally floating shield [3], will be called the 3 -body model, since the temperature rise of the shield is also a parameter of interest. The mathematical description of the temperature rises for the 3-body instrument is considerably more cumbersome than that for the 2-body instrument, but the rewards gained by operating with a thermally floating shield make it well worth while.

The following description assumes that each of the three calorimeter components is free of temperature gradients and that all heat transfer coefficients and heat capacities are constant. It deals with the case where the input power is constant, but can also apply when the power fluctuates with time [1-3].

Let $T_{1}, T_{2}, T_{3}$ represent temperature rises $(\mathrm{K})$ of the core, jacket, and shield, respectively, above the constant temperature of the surrounding medium,

$C_{1}, C_{2}, C_{3}$ represent the heat capacities $(\mathrm{J} / \mathrm{K})$ of the core, jacket, and shield, respectively (with $C_{1}=C_{2}$ ),

$P_{1}, P_{2}, P_{3}$ represent the constant power (W) applied to the core, jacket, and shield, respectively, and

$K_{1}, K_{2}, K_{3}$ represent the heat transfer coefficient $(\mathrm{W} / \mathrm{K})$ between the core and jacket, jacket and shield, and shield and medium, respectively.

The differential eqs of heat flow in the 3-body calorimeter are:

$$
\begin{aligned}
& P_{1} d t=C_{1} d T_{1}+K_{1}\left(T_{1}-T_{2}\right) d t, \\
& P_{2} d t=C_{2} d T_{2}-K_{1}\left(T_{1}-T_{2}\right) d t+K_{2}\left(T_{2}-T_{3}\right) d t,
\end{aligned}
$$

and

$$
P_{3} d t=C_{3} d T_{3}-K_{2}\left(T_{2}-T_{3}\right) d t+K_{3} T_{3} d t,
$$

where $t$ is time. The general solutions of these eqs are of the form: 


$$
T_{i}(t)=\sum_{j=1}^{3} \beta_{i j} e^{-r_{j} t}+T_{i}(\infty),
$$

where $i$ can be 1,2 , or 3 . The quantities $r_{1}, r_{2}$, and $r_{3}$ are roots of the eq:

$$
r^{3}-l r^{2}+m r-n=0,
$$

where:

$$
\begin{aligned}
l & =\frac{K_{1}}{C_{1}}+\frac{K_{1}+K_{2}}{C_{2}}+\frac{K_{2}+K_{3}}{C_{3}}, \\
m & =\frac{K_{1} K_{2}}{C_{1} C_{2}}+\frac{K_{1} K_{2}+K_{1} K_{3}}{C_{1} C_{3}}+\frac{K_{1} K_{2}+K_{1} K_{3}+K_{2} K_{3}}{C_{2} C_{3}},
\end{aligned}
$$

and

$$
n=\frac{K_{1} K_{2} K_{3}}{C_{1} C_{2} C_{3}}
$$

The asymptotic temperature rises are:

$$
\begin{aligned}
& T_{1}(\infty)=\frac{P_{1}}{K_{1}}+\frac{P_{1}+P_{2}}{K_{2}}+\frac{P_{1}+P_{2}+P_{3}}{K_{3}}, \\
& T_{2}(\infty)=\frac{P_{1}+P_{2}}{K_{2}}+\frac{P_{1}+P_{2}+P_{3}}{K_{3}},
\end{aligned}
$$

and

$$
T_{3}(\infty)=\frac{P_{1}+P_{2}+P_{3}}{K_{3}} .
$$

The coefficients are:

$\beta_{i 1}=\frac{\left(r_{2}-r_{3}\right)}{\Delta}\left[r_{2} r_{3} \gamma_{i}+\left(r_{2}+r_{3}\right) \alpha_{i}+Q_{i}\right]$,

$\beta_{i 2}=\frac{\left(r_{3}-r_{1}\right)}{\Delta}\left[r_{3} r_{1} \gamma_{i}+\left(r_{3}+r_{1}\right) \alpha_{i}+Q_{i}\right]$,

and

$\beta_{i 3}=\frac{\left(r_{1}-r_{2}\right)}{\Delta}\left[r_{1} r_{2} \gamma_{i}+\left(r_{1}+r_{2}\right) \alpha_{i}+Q_{i}\right]$,

where:

$$
\begin{aligned}
\Delta & =r_{2} r_{3}\left(r_{2}-r_{3}\right)+r_{3} r_{1}\left(r_{3}-r_{1}\right)+r_{1} r_{2}\left(r_{1}-r_{2}\right), \\
\gamma_{i} & =T_{i}(0)-T_{i}(\infty), \\
\alpha_{1} & =\frac{1}{C_{1}}\left\{P_{1}-K_{1}\left[T_{1}(0)-T_{2}(0)\right]\right\}, \\
\alpha_{2} & =\frac{1}{C_{2}}\left\{P_{2}+K_{1}\left[T_{1}(0)-T_{2}(0)\right]-K_{2}\left[T_{2}(0)\right.\right. \\
& \left.\left.-T_{3}(0)\right]\right\},
\end{aligned}
$$

$\alpha_{3}=\frac{1}{C_{3}}\left\{P_{3}+K_{2}\left[T_{2}(0)-T_{3}(0)\right]-K_{3} T_{3}(0)\right\}$,

$Q_{1}=\frac{1}{C_{1}}\left[-K_{1}\left(\alpha_{1}-\alpha_{2}\right)\right]$,

$Q_{2}=\frac{1}{C_{2}}\left[K_{1}\left(\alpha_{1}-\alpha_{2}\right)-K_{2}\left(\alpha_{2}-\alpha_{3}\right)\right]$,

and

$Q_{3}=\frac{1}{C_{3}}\left[K_{2}\left(\alpha_{2}-\alpha_{3}\right)-K_{3} \alpha_{3}\right]$.

The temperature-time curves shown in figures 2 and 3 were calculated from these eqs using the experimental values of heat capacities and transfer coefficients for one of the calorimeters described in this report. The exposure times chosen are typical of times encountered with two different radiation sources in this laboratory, a linear accelerator (100 s exposures) and a 500 -curie cobalt- 60 source (1000 s exposures).

In each of figures 2 and 3 , the upper curve was calculated for an irradiation, assuming a uniform dose

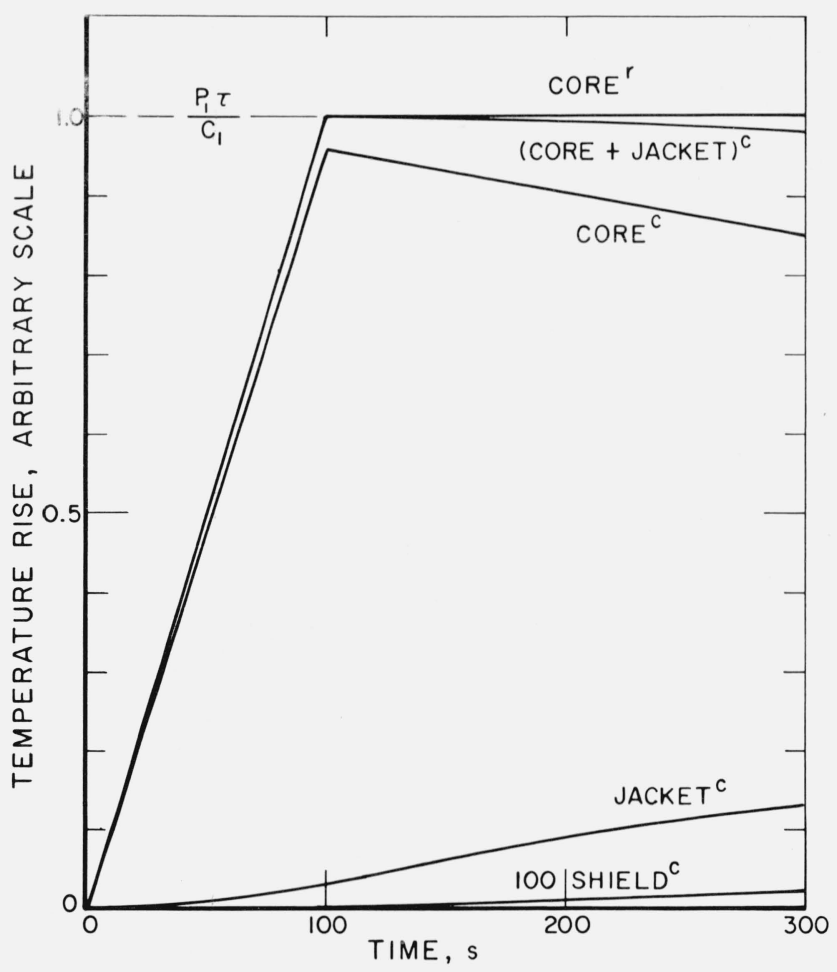

FIGURE 2. Relative temperature-time behavior of the three-body calorimeter for $100 \mathrm{~s}$ of heating.

The top curve (superscript $r$ ) shows the core response for a uniform dose rate throughout the calorimeter. The other curves (superscript $c$ ) show its behavior when heat is applied only to the core. The temperature scale is normalized to $P_{1} \tau / C_{1}=1$, where $\tau$ is the heating time. 


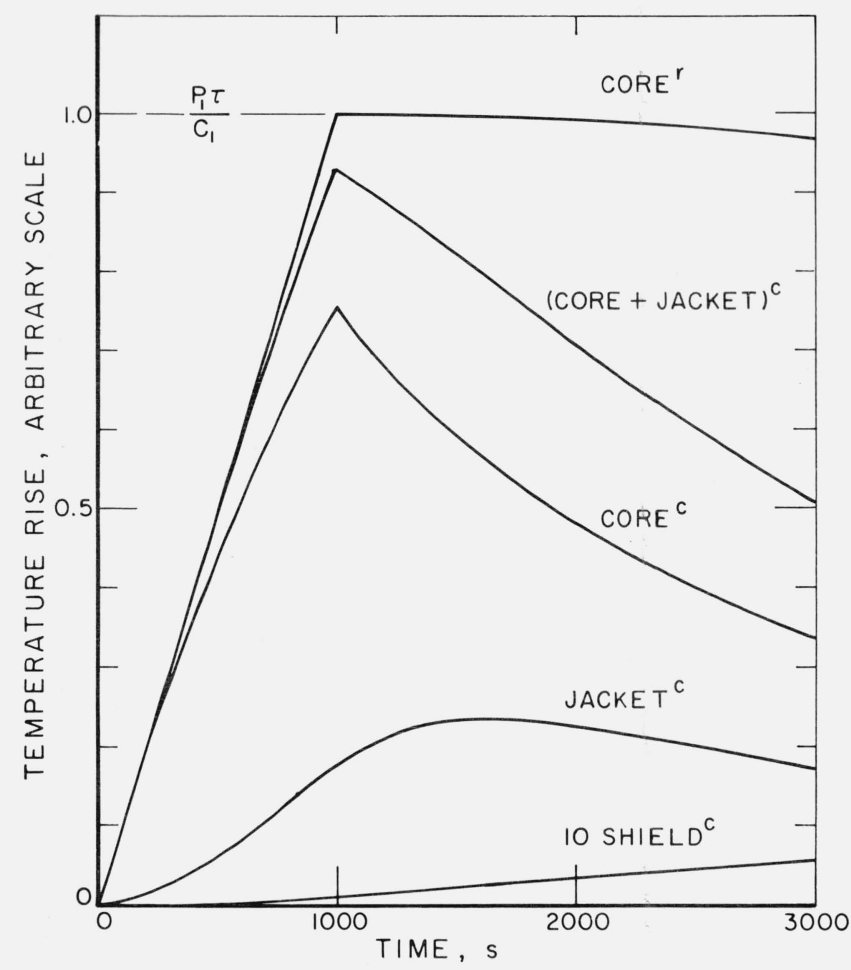

FIGURE 3. Relative temperature-time behavior of the three-body calorimeter for 1000 s of heating.

rate throughout the three bodies (so that $P_{1} / C_{1}=P_{2} /$ $\left.C_{2}=P_{3} / C_{3}\right)$. The other curves all refer to electrical calibrations, with power $P_{1}$ supplied to the core alone $\left(P_{2}=P_{3}=0\right)$. In both the irradiation and calibration cases, the core temperature rises to a maximum, and decreases after power is turned off. In the radiation case, the jacket and shield temperatures behave in the same manner, but their temperature rises are slightly smaller. They are not shown in figures 2 and 3 for the sake of clarity. In the calibration case, the jacket and shield temperatures rise more slowly, since these components are not heated directly, and continue to rise for some time after power is turned off.

The core curves of figures 2 and 3 can be observed experimentally by following the output voltage of the circuit of figure 4, an equal-arm Wheatstone bridge. Similarly, the core-plus-jacket curves can be observed by following the output voltage of the bridge of figure 5 , which includes the jacket thermistor.

The input energy during these experiments can be evaluated by integration of eqs (1) and (2). For measurements with the circuit of figure 4 , integration of (1) shows that with $T_{1}(0)=0$, the energy supplied to the core during a run of duration $\tau$ can be expressed as:

$$
E_{C}=\int_{0}^{\tau} P_{1} d t=C_{1} T_{1}(\tau)\left(1+F_{C}\right),
$$

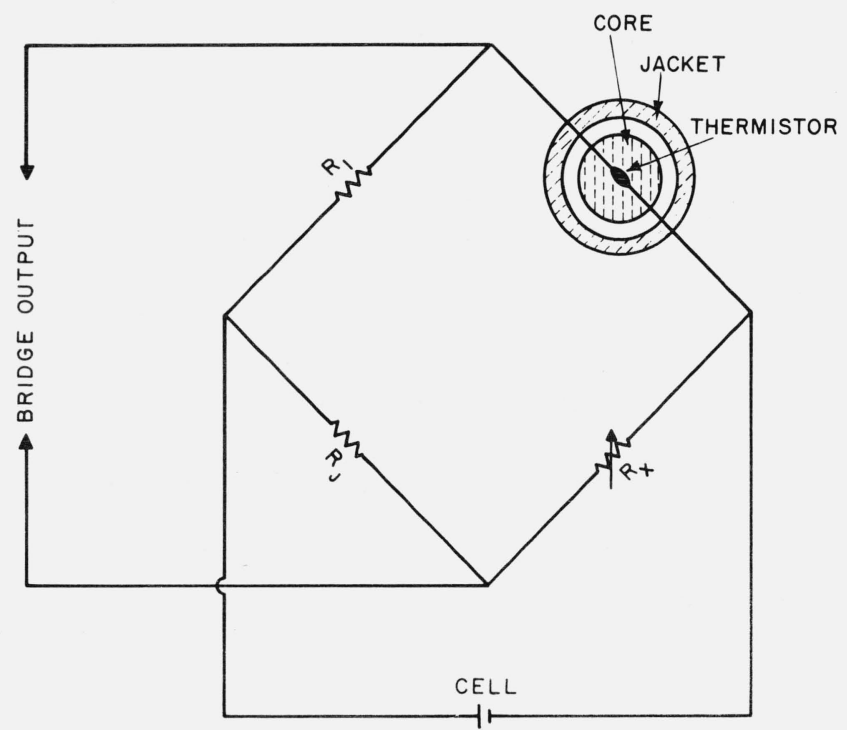

FIGURE 4. Circuit for measuring the core temperature rise.

where:

$$
F_{C}=\frac{K_{1} \int_{0}^{\tau}\left(T_{1}-T_{2}\right) d t}{C_{1} T_{1}(\tau)} .
$$

Equation (23) gives the core energy for either an irradiation $\left(E_{C}^{r}\right)$ or a calibration $\left(E_{C}^{c}\right)$, although the correction factor for the former is much smaller than for the latter. Calculated values of $F_{C}^{r}$ and $F_{C}^{c}$ for times up to $1000 \mathrm{~s}$ are shown in figure 6, using the same C's and K's used to obtain the curves of figures 2 and 3 .

The correction factor for calibrations can be reduced considerably by using the circuit of figure 5 . For this

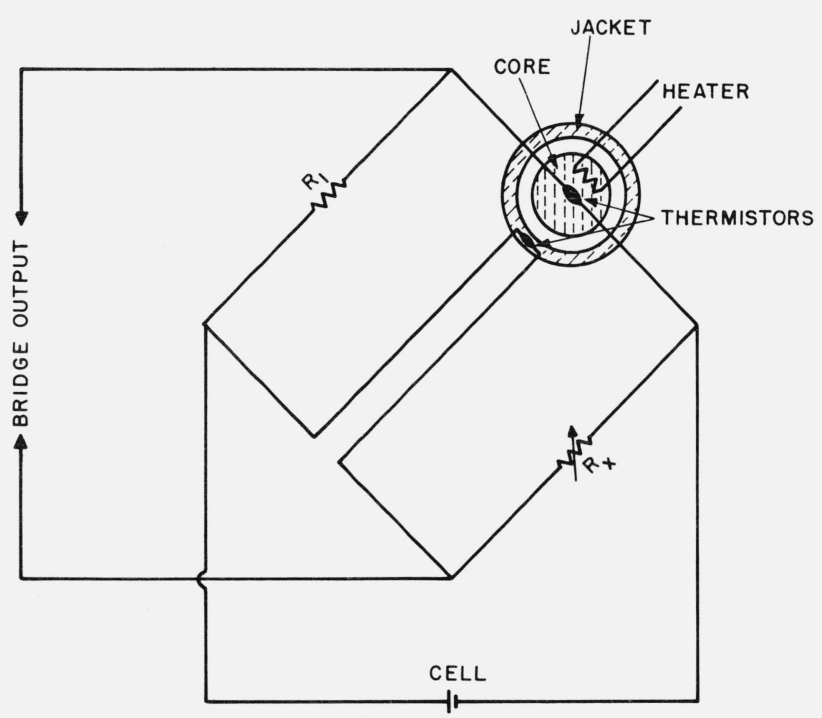

FIGURE 5. Circuit for measuring the core-plus-jacket temperature rise. 
case, with $P_{2}=P_{3}=0$, integration of the sum of eqs (1) and (2) shows that the electrical energy supplied to the core, can be expressed as:

$E_{C+J}^{c}=\int_{0}^{\tau} P_{1} d t=C_{1}\left[T_{1}(\tau)+T_{2}(\tau)\right]\left(1+F_{C+J}^{c}\right)$,

where:

$$
F_{C+J}^{c}=\frac{K_{2} \int_{o}^{\tau}\left(T_{2}-T_{3}\right) d t}{C_{1}\left[T_{1}(\tau)+T_{2}(\tau)\right]} .
$$

Calculated values of $F_{C+J}^{c}$ for times up to $1000 \mathrm{~s}$ are also shown in figure 6 . Note that the use of both

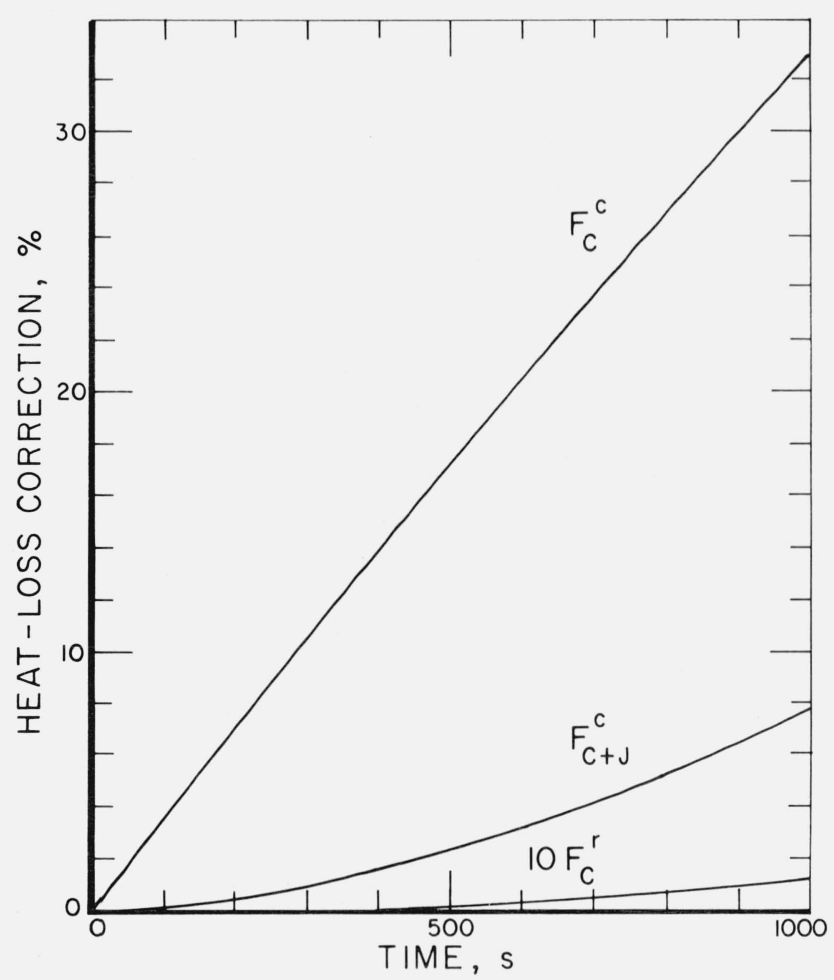

FigURE 6. Calculated heat loss correction vs. duration of run.

core and jacket thermistors reduces the size of the correction $^{4}$ by a large factor, and thus in practice it may decrease the uncertainty of the heat-loss correction.

If radiation runs in the 1-thermistor mode are compared with calibration runs in the 2-thermistor mode, the radiation energy absorbed in the core can be determined from the equation:

\footnotetext{
${ }^{4}$ This correction can be determined at any time. Then at any time, $t$, after power turn-off, $\left[T_{1}(t)+T_{2}(t)\right]\left(1+F_{C+J}^{c}\right)$ is a constant, after the thermal gradients inside the core have
} disappeared.

$$
E_{C}^{r}=E_{C+J}^{c}\left[\frac{T_{1}^{r}\left(\tau^{r}\right)}{T_{1}^{c}\left(\tau^{c}\right)+T_{2}^{c}\left(\tau^{c}\right)}\right]\left[\frac{1+F_{C}^{r}}{1+F_{C+J}^{c}}\right],
$$

using the measured calibration energy, measured temperature rises, and calculated heat-loss corrections. ${ }^{5}$ The absorbed dose in the core is then

$$
D=100 \frac{E_{C}^{r}}{M_{C}} \mathrm{rad},
$$

where $M_{C}$ is the core mass in $\mathrm{kg}$ if $E_{C}^{r}$ is in joules.

There is an interesting relation between the core temperature rise during and after a radiation measurement, $T_{1}^{r}$, and the temperature rises of the bodies during and after a calibration, $T_{1}^{c}, T_{2}^{c}$, and $T_{3}^{c}$. If the same power is applied to the core for the same length of time in each case, it can be shown that for any value of $t$ :

$$
T_{1}^{r}(t)=T_{1}^{c}(t)+T_{2}^{c}(t)+\frac{C_{3}}{C_{1}} T_{3}^{c}(t) \cdot
$$

This is a special case of a more general theorem which was discussed in the early references [1-3], using a slightly different notation. In addition, that theorem also shows the equivalence of quasi-adiabatic calibrations and heat-loss compensated calibrations for a 3-body calorimeter. In a quasi-adiabatic calibration, $P_{1}=P_{2}$ and $P_{3}=0$, and the temperature rise of the core, $T_{1}^{q}(t)$, is identical with the sum of the core and jacket temperature rises when the core alone is heated with power $P_{1}$ :

$$
T_{1}^{q}(t)=T_{1}^{c}(t)+T_{2}^{c}(t) .
$$

This equation also applies to a 2-body calorimeter.

\section{Sources of Systematic Error}

The theory of section 2 assumed that each calorimeter component was free of temperature gradients at all times. In practice, this is never completely true, which raises several questions. Is the thermistor in each component located in a position where it will correctly indicate the average temperature of that component? Again, if there are temperature variations along the surface of any component which affect the average rate of heat loss, are eqs (1) to (3) an adequate description of the calorimeter operation? Differences of the order of 1 percent across the surface of a 2 piece core of quasi-adiabatic construction have been reported [9]. Whether or not such gradients affect the accuracy of the calorimeter measurements is a complicated question, depending on exact details of calorimeter construction and operation.

${ }^{5}$ Note that since $\mathbf{E}_{C}^{r}$ is determined from ratios, it is independent of both $C_{1}$, except in a
minor way in the calculated corrections, and the absolute calibration of the temperature minor
scale. 
During irradiation runs, energy is deposited in proportion to absorbed dose throughout the calorimeter, ${ }^{6}$ and the thermal gradients are quite small. Much larger thermal gradients appear during calibration runs, when electrical power is generated in the core alone. Attempts are usually made to distribute the source of calibration power as evenly as possible throughout the core [7-9] to minimize these gradients. In the present case, this was not done, and calibration power was generated in a small spherical heater (a thermistor) attached to the core near its surface. One would expect the effects, if any, of these gradients to be considerably reduced by use of the two-thermistor bridge instead of the single-thermistor bridge. The jacket acts as a thermal buffer and heat retainer which in effect allows more time for large thermal gradients in the core to dissipate. Thus, heat loss from the system is not from a non-uniform temperature core, but from the jacket, which has a lower and more uniform temperature.

After calibration power is turned off, and thermal gradients around the core heater have disappeared, there will be a radial temperature drop because of thermal leakage to the jacket. This tends to reduce the temperature of the core thermistor, which is located close to the surface, where the temperature is lower than the average core temperature. This could lead to a systematic error if calibrations were performed with the single-thermistor bridge. This error will be at least partially cancelled by using the two-thermistor bridge, because the jacket thermistor, close to the inner surface of the jacket, will be raised above the average jacket temperature because of heat flow from the core.

The core thermistor leads conduct heat from the core thermistor, leading to erroneous thermistor readings and systematic error. This error can normally be made negligible by using long leads of small diameter, but even in applications where this would be difficult, the error can be effectively eliminated in a two-thermistor bridge. This is done by using leads of equal length for core and jacket thermistors, and thermally attaching the core leads to the jacket and the jacket leads to the core, as shown in figure 7. Any reduction of the core thermistor temperature is compensated by an increase in the jacket thermistor temperature.

Another source of systematic error is thermal conduction along the heater leads. These leads conduct more heat from the core during calibrations than during irradiations, because in the former case, the heater is at a higher temperature. Again, this error can be made negligible by using long, thin leads, but even where this cannot be done, most of the heat lost from the core can be retained in the jacket of a twothermistor calorimeter if the heater leads are thermally tied to the jacket. ${ }^{7}$

The four systematic errors discussed in the last four paragraphs should all be reduced in size by changing

\footnotetext{
${ }^{6}$ Absorbed dose varies with depth in any material, in a manner which depends on the energy spectrum of the incident radiation.

${ }^{7}$ In the present calorimeters, the errors discussed in the last two paragraphs were minimized by using long leads, which were not attached to the jacket. However, heat radiated from the leads is still measured.
}

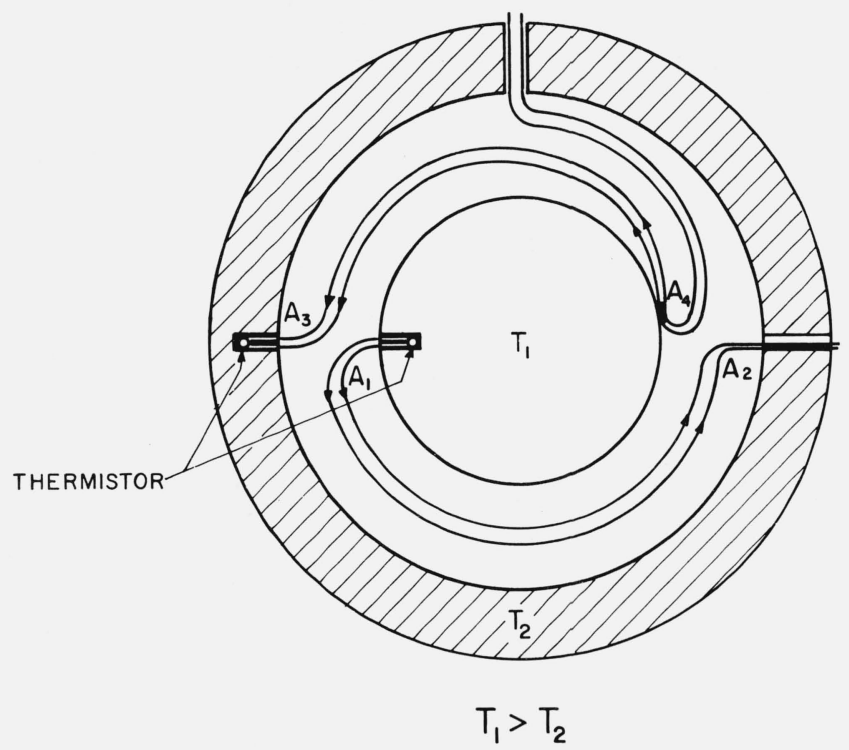

FigURE 7. Illustration of heat flow caused by temperature gradients along the sensor leads.

When the length of $A_{1} A_{2}$ equals the length of $A_{3} A_{4}$, the cooling effects of the core sensor leads are in principle compensated by the heating effects of the jacket sensor leads.

from the single-thermistor bridge to the two-thermistor bridge for calibration runs. This suggests a test for the combined magnitude of these effects. By comparing corrected temperature rises in identical calibration runs in the two modes, it should be possible to check whether $E_{C}^{c}$ from (23) is the same as $E_{C+J}^{c}$ from (25). Any discrepancy would indicate that these systematic errors are large enough to be measurable.

A second consistency check can be performed by testing the equality of quasi-adiabatic calibration and heat-loss-compensated calibration, discussed at the end of section 2. The detection of any failure of the equality of eq (30) would be an indication that some of these systematic errors are appreciable.

\section{Calorimeter Construction}

Two carbon calorimeters have been constructed from high-purity reactor-grade graphite $\left(\rho=1.70 \mathrm{~g} / \mathrm{cm}^{3}\right)$ and are now in operation at the National Bureau of Standards. They have identical core-jacket-shield assemblies and differ only in the dimensions of the temperature-controlled medium surrounding the shield. The large model, which is not portable, is permanently mounted where it can be irradiated with electron and photon beams from the NBS $100-\mathrm{MeV}$ linear accelerator (linac). The small model, which is portable, will be described in detail.

The construction of the calorimeters was undertaken with attention to good conventional calorimetric technique, in the expectation that they would perform 
well even without the advantages of heat-loss compensation discussed in the preceding section. This section describes a number of constructional details that are generally applied in making an accurate, reliable, and stable calorimeter. However, some constructional details were influenced by special problems encountered in the measurement of absorbed dose. Therefore, details of the final design of the calorimeter and its circuit were necessarily based on a number of compromises. Tests and performance indicate that their design and fabrication are satisfactory.
Figure 8 is a schematic cross section of the portable calorimeter, and figure 9 is a photograph of the beam exit side with the rear plates and the core-jacket-shield assembly removed. Figures 10 to 12 are photographs of the assembly with the shield cap and jacket cap removed.

The core consists of a single graphite disc, $20 \mathrm{~mm}$ in diameter and $2.75 \mathrm{~mm}$ thick. It contains two $0.25 \mathrm{~mm}$ (0.010-in) diameter thermistors, embedded $2 \mathrm{~mm}$ deep in $0.33-\mathrm{mm}(0.013-\mathrm{in})$ diameter holes filled with cement. One of these thermistors has a resistance of $1500 \Omega$
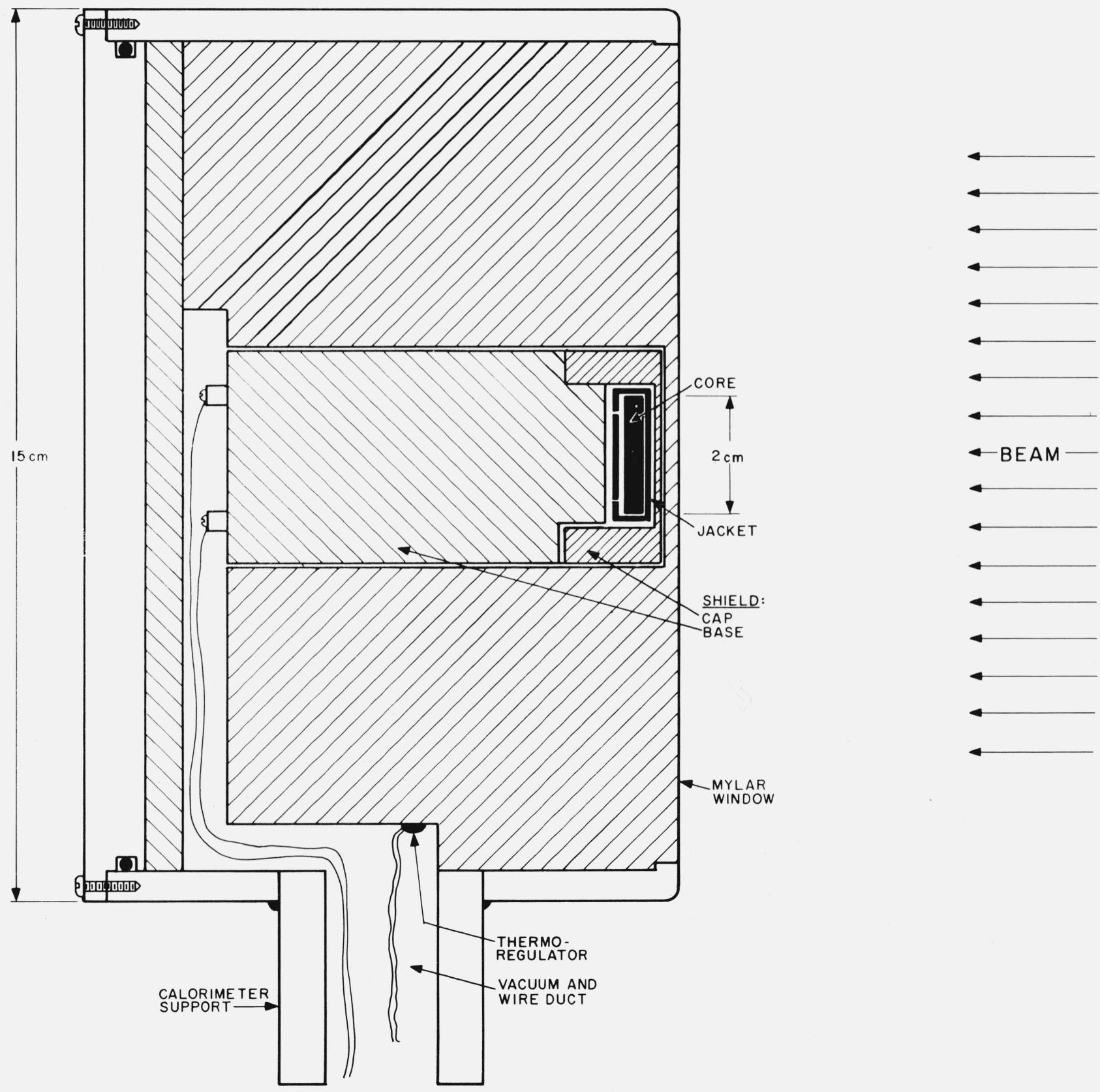

FIGURE 8. Side-view cross section of the assembled portable calorimeter. 

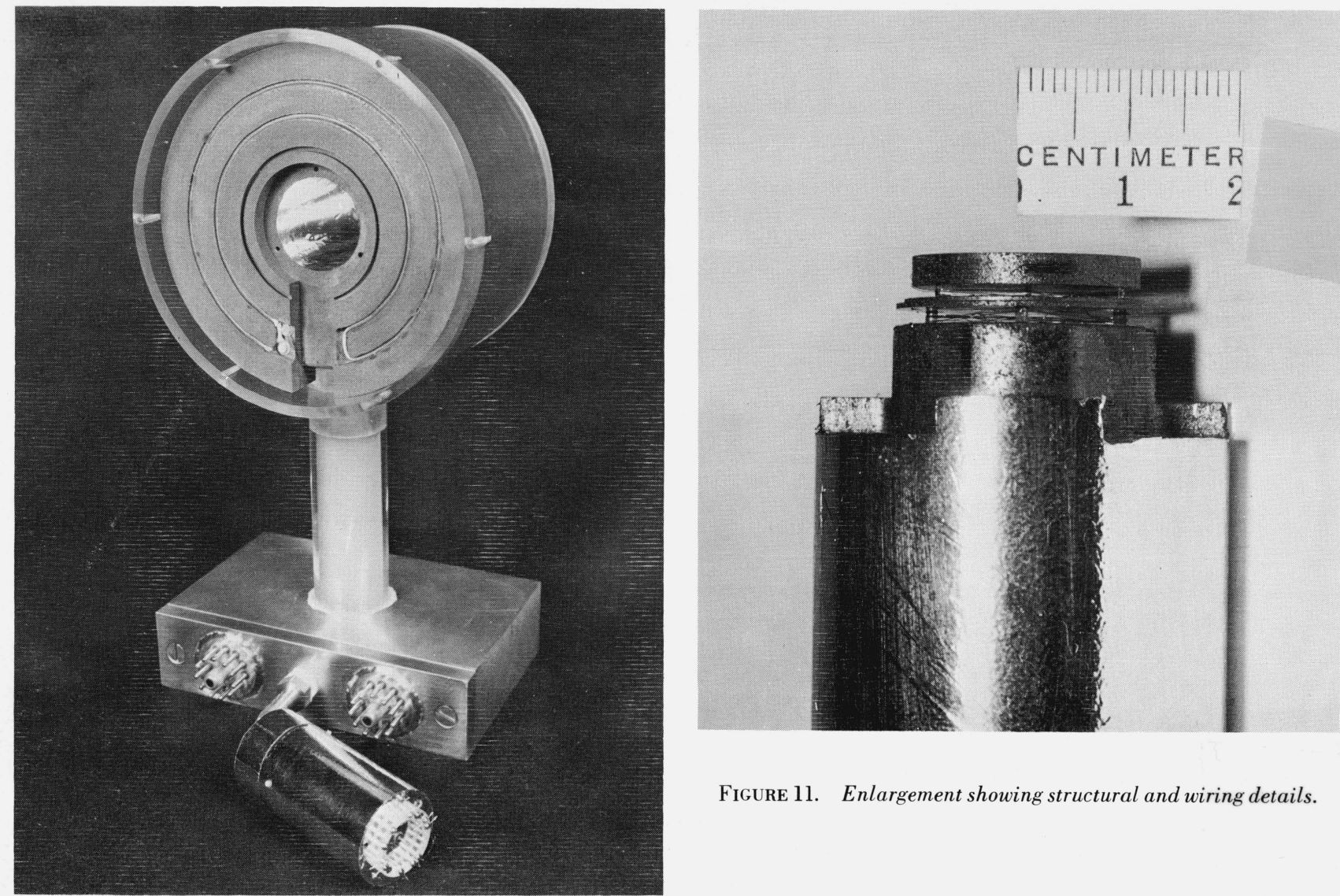

FIGURE 11. Enlargement showing structural and wiring details.

FIGURE 9. Rear-view photograph of the portable calorimeter showing the removed core-jacket-shield assembly.
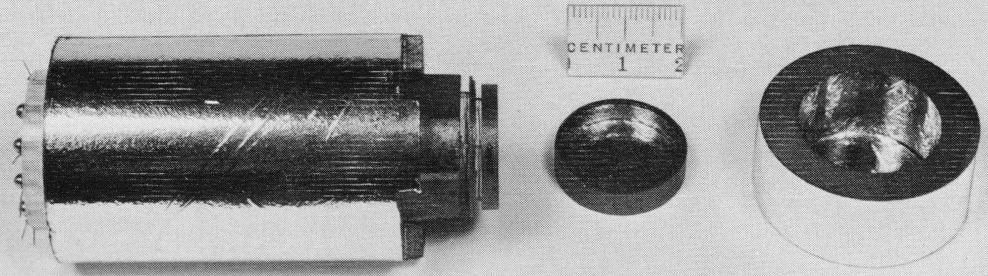

Figure 10. Constructional details of the core-jacket-shield assembly, with the core and jacket caps removed. 


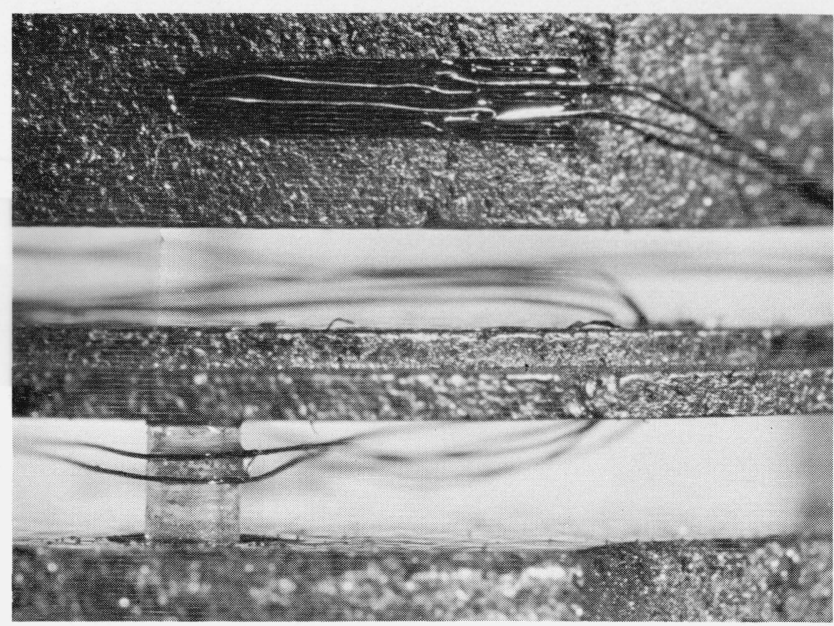

FIGURE 12. Further enlargement showing embedment of core thermistor, wiring, jacket base, and a jacket base support $(1 \mathrm{~mm}$ dia) mounted on the shield.

(at $303 \mathrm{~K}$ ) and is used in the bridge circuits of figures 4 and 5 to indicate temperature. The other thermistor has a resistance of $20,000 \Omega$ and is used as the calibration heater. The bare platinum leads of each of these thermistors were cemented to the core for a length of about $4 \mathrm{~mm}$, using a thin layer of plastic for electrical insulation. The thermistor leads had been soldered to \# 44 enameled copper wires, $0.05 \mathrm{~mm}$ (0.002 in) in diameter, as shown in figure 12. An additional lead, $0.015 \mathrm{~mm}(0.0006 \mathrm{in})$ in diameter and constructed of an alloy with low thermal conductivity, was provided for electrically grounding the core to a point external to the calorimeter.

The core was mounted on the base of the 2-piece jacket with three polystyrene supports, $0.5 \mathrm{~mm}$ in diameter and $1 \mathrm{~mm}$ long. The materials enclosed by the jacket are listed in table 1, which shows that the weight of impurities is less than 0.4 percent of the total weight of the core. An estimate of the difference of the heating effects from that of carbon during beam irradiation indicates an insignificant effect $(<0.1 \%)$ on a measurement.

TABLE 1. Portable calorimeter: composition of core, supports, and wiring

\begin{tabular}{|c|c|}
\hline Material & Mass (mg) \\
\hline Core, carbon.............. & 1416.0 \\
\hline Lead insulation, mylar................ & 0.2 \\
\hline Cement.............................. & 0.7 (est.) \\
\hline Core supports, polystyrene........................... & 1.3 \\
\hline Thermistors, core ................................... & 0.04 \\
\hline $\begin{array}{l}\text { Leads between core and jacket (for thermistor, } \\
\text { heater, and ground) } \ldots \ldots \ldots \ldots \ldots \ldots \ldots \ldots \ldots \ldots \ldots \ldots \ldots \ldots \ldots \ldots \ldots \ldots\end{array}$ & 3. \\
\hline
\end{tabular}

The inside dimensions of the jacket are a diameter of $21 \mathrm{~mm}$ and a width of $4.15 \mathrm{~mm}$. The inner surfaces are lined with grounded aluminized mylar, $0.006 \mathrm{~mm}$ $(0.00025$ in $)$ thick. Separate tests with this reflecting material showed that it was much more effective in retarding heat transfer than was aluminum evaporated directly onto graphite. The mass of the aluminized mylar and the adhesive with which it was attached was about 3 percent of the mass of the jacket.

The outside dimensions of the jacket were determined by the requirement that jacket and core have equal heat capacities. The core and jacket were not assembled until the masses of all the components had been determined. The jacket was deliberately made slightly oversize, and it was trimmed by hand until the calculated sum of the heat capacities of the individual jacket components was equal to a similar sum of the core component heat capacities.

A $1500-\Omega$ thermistor was embedded with cement in the jacket base, $0.9 \mathrm{~mm}$ thick. Its platinum leads were cemented to the inner surface of the jacket. A length of about $5 \mathrm{~cm}$ of the copper leads from this thermistor and from the two core thermistors was left in the void between core and jacket, wound around and cemented to the polystyrene core supports. These, along with the core grounding wire, emerge from the jacket through a hole required for pump-down and are there joined by a second grounding wire attached to the jacket.

The jacket cap was tightly fitted to the jacket base and lightly bonded with cement. Tests were performed which showed that there is good thermal contact between cap and base. ${ }^{8}$ The jacket is mounted on the shield with three 1-mm diameter polystyrene supports, which are recessed into enlarged holes in the shield. The thermal path length is thus $6 \mathrm{~mm}$ although the jacket base and shield are separated by only $1.25 \mathrm{~mm}$.

The external dimensions of the shield are a diameter of $36 \mathrm{~mm}$ and a length of $74 \mathrm{~mm}$. The shield is 85 times as massive as the core; it has ample heat capacity to protect the core from external temperature fluctuations transmitted by the 2-mm-thick front surface of the medium. The front of the shield cap is only $1 \mathrm{~mm}$ thick. The cap is firmly fiitted onto the shield base and sealed with a graphite colloidal suspension. All of the surfaces of the shield are covered with aluminized mylar.

The thermistor leads emerging from the jacket are wound around and cemented to the jacket supports, as shown in figure 11, for a length of about $5 \mathrm{~cm}$ before passing to the shield. They are cemented to the shield for a length of about $1 \mathrm{~cm}$ and then pass through a pump-out hole to the terminals shown in figures 9 and 10.

The graphite medium was machined from the same graphite block from which the core, jacket, and shield were made. Its internal surfaces, facing the shield, are

${ }^{8}$ Practical considerations, including the geometry of the jacket, allowed only a rough approximation to a further constructional advantage, which is to construct each piece with a mass proportional to the heat received from the core. This would have provided even more assurance of temperature uniformity of the jacket. 
all covered with aluminized mylar. The radial and axial spacings between shield and medium are $1 \mathrm{~mm}$. The medium rests in a cylindrical plastic vacuum box whose front surface consists of a $0.13-\mathrm{mm}(0.005$-in) thick mylar window which presses directly upon the medium itself. The outside dimensions of this box are 6 in in diameter and 4 in in length. The temperature 1 of the medium is stabilized at $303 \mathrm{~K}$ by a thermoregulator. The position of the temperature sensing element of this system is shown in figure 8 , and the heating coils (wires) are indicated in figure 9.

The circuit shown in figure 13 is used to measure calorimeter temperature changes during both calibration and radiation runs, and to return the calorimeter

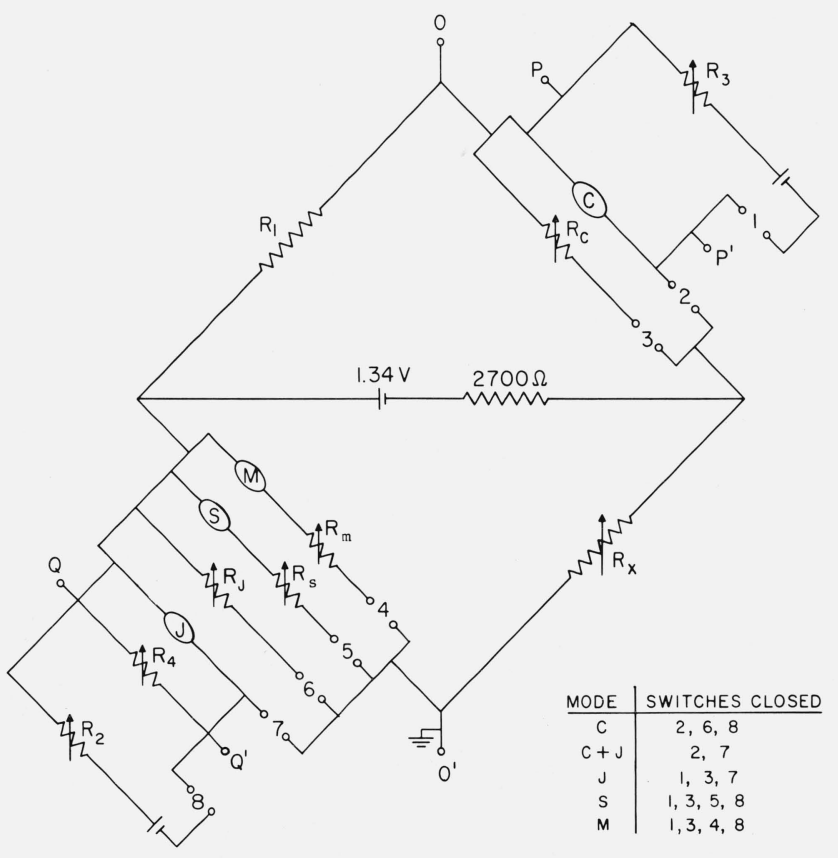

FIGURE 13. Measurement and control circuit.

The circuit makes possible rapid restoration of the calorimetric bodies to equilibrium temperatures.

components to equilibrium after each run. The circuit consists of an equal-arm Wheatstone bridge using one or more of the four $1500-\Omega$ thermistors C, J, S, and M (in the core, jacket, shield, and medium, respectively). There is a five-position ganged switch for connecting any of these four thermistors in the bridge, or for connecting $\mathrm{C}$ and $\mathrm{J}$ in opposite arms. The bridge output voltage developed between points $\left(0,0^{\prime}\right)$ is amplified, displayed on a galvonometer, and simultaneously recorded on a paper chart.

Calibration runs are made in the $\mathrm{C}+\mathrm{J}$ mode, with $\mathrm{C}$ and $\mathrm{J}$ in opposite arms of the bridge, and with the fixed $1500-\Omega$ resistor $R_{1}$ and the variable resistor $R_{x}$ (a $1700-\Omega$ fixed resistor shunted with a $10-\mathrm{k} \Omega$ decade resistor box) in the other two arms (fig. 5). For beam measurements, in the $\mathrm{C}$ mode, thermistor $\mathrm{J}$ is replaced by a resistor $R_{J}$ of equal resistance (fig. 4), and an auxiliary circuit maintains $\mathbf{J}$ at its equilibrium temperature. There is also an auxiliary circuit to maintain thermistor $\mathrm{C}$ at its equilibrium temperature when, in measurement mode $\mathrm{J}, \mathrm{S}$, and $\mathrm{M}$, it is replaced by resistor $R_{C}$ in the bridge circuit.

Thermistors $\mathrm{S}$ and $\mathrm{M}$ were found to have slightly smaller resistances than did thermistors $\mathrm{C}$ and $\mathrm{J}$, and resistors $R_{s}$ and $R_{m}$ were added to compensate for the difference. Normally about $55 \mathrm{ohms}$, they are adjusted so that the switch can be changed from $\mathrm{C}$, $\mathrm{J}$, or $\mathrm{C}+\mathrm{J}$ mode to the $\mathrm{S}$ or $\mathrm{M}$ mode without disturbing the balance of the circuit. ${ }^{9}$ Resistance $R_{4}$ is a $600-\mathrm{k} \Omega$ fixed resistor in series with a $300-\mathrm{k} \Omega$ variable resistor, whose purpose is to permit small changes to be made in the bridge balance in the $\mathrm{C}+\mathrm{J}$ mode, as explained in the following section.

The power supplied to the core heater during a calibration run is measured with a potentiometer at points approximately $30 \mathrm{~m}(100 \mathrm{ft})$ from the calorimeter. The heater current is determined by measurement of the potential difference across a fixed resistor of accurately known resistance (nominally $20 \mathrm{k} \Omega$ ), in series with the heater. The potential difference across the heater and its leads is also measured, and gives the heater voltage after a correction is made for potential drop in the leads. This correction included all of the leads up to a point in the void between jacket and shield, but only amounts to about 0.02 percent of the heater voltage.

\section{Measurement, Analysis, and Control Techniques}

The measurement and control circuit of figure 13 is used as follows. Start with the calorimeter components at their equilibrium temperatures ${ }^{10}$ in the $\mathrm{C}+\mathrm{J}$ mode (fig. 5). Adjust $\boldsymbol{R}_{x}$ to get zero bridge output, and call this $R_{x}(0)$. Measure the voltages across the $\mathrm{C}$ thermistor (at points $\mathrm{P}$ and $\mathrm{P}^{\prime}$ ) and across the $\mathrm{J}$ thermistor (at points $\mathrm{Q}$ and $\mathrm{Q}^{\prime}$ ), and call these equilibrium voltages $V_{C}$ and $V_{J}$, respectively. Switch to mode C (fig. 4), adjust $R_{J}$ until the bridge output is again zero, and adjust $R_{2}$ until the voltage between $\mathrm{Q}$ and $\mathrm{Q}^{\prime}$ is again equal to $V_{J}$. Switch to mode J, adjust $R_{C}$ to get zero bridge output, and adjust $R_{3}$ to get voltage $V_{C}$ between $\mathrm{P}$ and $\mathrm{P}^{\prime}$. Switch to mode $\mathrm{S}$, and adjust $R_{s}$ to get zero bridge output. Switch to mode $\mathrm{M}$, and adjust $R_{m}$ to get zero bridge output. After these steps have been completed, the switch

\footnotetext{
${ }^{9}$ There is no need for an auxiliary circuit to heat thermistor $\mathrm{M}$ when it is not in the bridge circuit, because of the large mass and temperature regulation of the medium. In the case of the less massive, unregulated shield, the circuit balance will be altered by prolonged use of thermistor $\mathrm{S}$, but this can be avoided by making only momentary observations of the shield temperature.

${ }^{10}$ The equilibrium temperatures of the different components differ because the 1.34-volt mercury bridge cell supplies about $38 \mu \mathrm{W}$ of power to each of the core and jacket thermis. tors. This produces extra terms in eqs (1) and (2), which disappear if $T_{i}$ is redefined as the temperature rise of the $i$ th component above its own equilibrium temperature. The justification for this is that the Wheatstone bridge measures precisely this redefined tem perature. The bridge power supplied to the thermistors does change during a run because perature The bridge power suppled to the thermistors does change during a run, because of the thermistor resistance changes, but by a negligible amount $(\ll 0.01 \%)$, compared
to input power.
} 


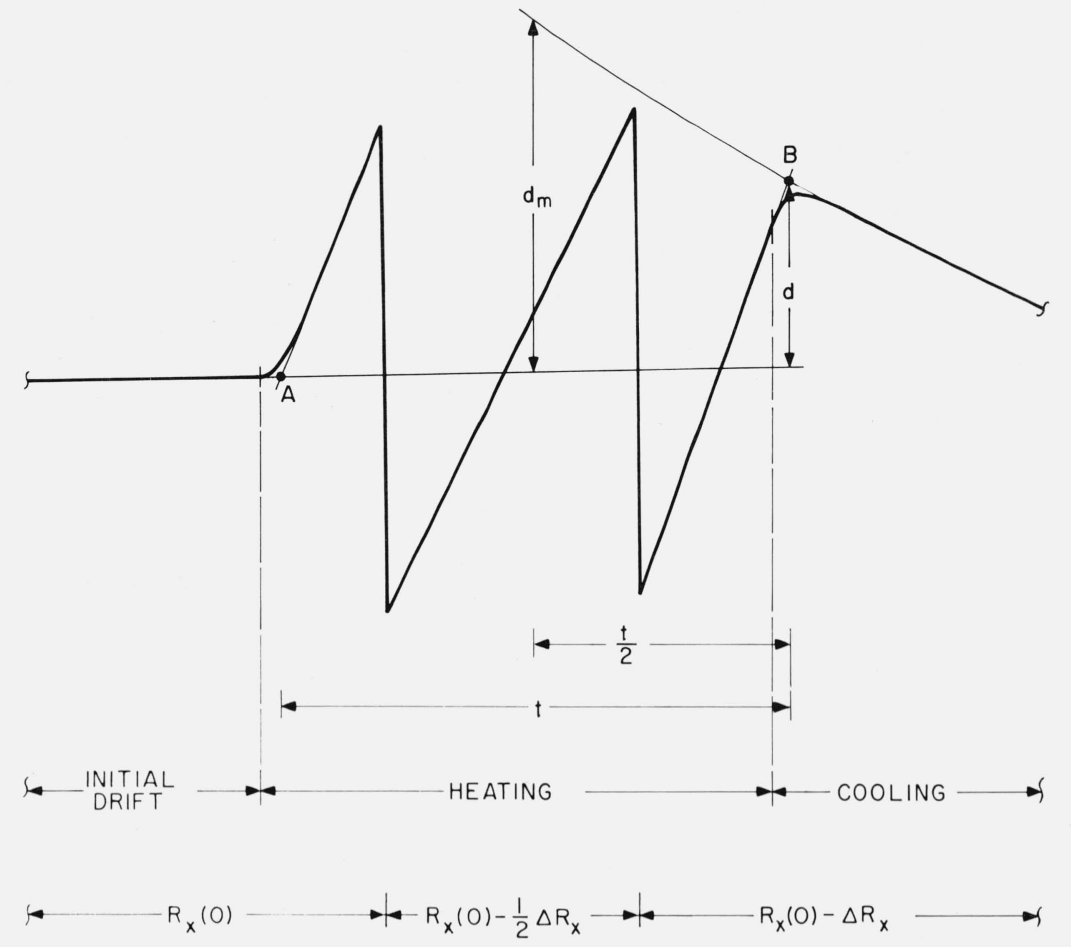

FIGURE 14. Schematic illustration of a chart record using the bridge null method of measurement, and of the empirical and analytical methods of correcting for heat loss.

can be changed to any position without changing the balance or disturbing equilibrium.

Next, make a calibration or a radiation run. Each run consists of three parts, an initial drift, with no input power, a heating curve, while power is on, and a cooling curve, after power has been turned off. The time allotted for the initial drift and the cooling curve will vary, but each should be at least as long as the heating curve, so that they can be accurately extrapolated, as discussed below.

For small temperature rises, the recorder chart pen deflection during the run will be small and there will be no need to change $R_{x}$. The precision of a measurement of the temperature rise in such a case is limited by the width of the recorder chart. Higher precision can be attained with larger temperature rises, using a null method of operation. As the temperature rises during the heating curve, $R_{x}$ is decreased to keep the pen on the chart, as indicated in the schematic example of figure 14. After completion of the cooling curve, the components can be restored to equilibrum, as described later in this section, in parparation for the next measurement.

There are two methods which have proven useful in evaluating the information in a chart run record. These will be called the analytical method, using calculated heat-loss corrections, and the empirical method, where the heat-loss corrections are de- termined graphically. The analytical method is useful only where the background drifts do not change noticeably during a given run, ${ }^{11}$ and where the input power is constant. The advantage of this method is that it provides an unbiased technique for correcting for heat loss, a technique which does not depend on the method chosen for extrapolation of a relatively non-linear and rapidly decaying cooling curve to the mid-run.

The analytical method requires measurement of the net pen displacement, $d$ (fig. 14). This is the vertical distance between point B (located by extrapolation of the heating and cooling curves, as shown in fig. 14), and an extrapolation of the small and essentially linear initial drift. The displacement, $d$, must be converted into resistance, using a conversion factor determined by making a known change in $R_{x}$ and observing the displacement produced. The fractional change in $R_{x}$ during the run, which is proportional to the observed temperature rise, is then $f=\left(\Delta R_{x}+d\right) / R_{x}(0)$. Then the input energy may be written:

$$
E=k f(1+F)
$$

${ }^{11}$ If the components were all in equilibrium, the initial drift rate would be zero. Obviously, the closer the system is to equilibrium, the smaller will be the change in drift during a run. 
where $k$ is a constant determined by calibration (see eqs (23) and (25)) and $F$ is a calculated heat-loss correction (fig. 6).

The empirical method does not require knowledge of the heat capacities and transfer coefficients, and tends to compensate for gradual changes in the initial drift rate. It requires measurement of the corrected net pen displacement, $d_{m}$ (fig. 14). This is the vertical distance between the extrapolated initial drift and cooling curves, midway between points $A$ and $B$. Extrapolation of the cooling curve is based on the approximation of the integrals in eqs (24) and (26) by:

$$
\begin{aligned}
& \int_{0}^{\tau} \Delta T(t) d t \\
& \quad=\frac{\Delta T(\tau)+\Delta T(0)}{2}\left(\tau+\epsilon_{1} \tau^{2}+\epsilon_{2} \tau^{3}+\ldots\right),
\end{aligned}
$$

where the coefficients $\epsilon_{1}$ and $\epsilon_{2}$ are zero for linear extrapolation. Again, $d_{m}$ must be converted into resistance, and the corrected fractional change in $R_{x}$, which is proportional to the corrected temperature rise, is $f_{m}=\left(\Delta R_{x}+d_{m}\right) / R_{x}(0)$.

Then, the input energy may be written:

$$
E=k f_{m},
$$

where $k$ is the same constant which appeared in eq (31), and which is to be evaluated in a calibration run.

The numbers in table 2 show how successfully the empirical method predicts the proper corrections for the examples given in figures 2 and 3 . The numbers are based on extrapolations of linear, quadratic, and cubic fits to the cooling curves between $t=\tau$ and $t=2 \tau$. It can be seen that linear extrapolation produces errors larger than 0.1 percent in all cases except the trivial case of irradiation for $100 \mathrm{~s}$, where the total correction is zero. Quadratic and cubic extrapolation, on the other hand, correctly predict the heat-loss corrections to within 0.1 percent for all cases with a 100 -s exposure

TABLE 2. Errors in heat-loss corrections obtained empirically with linear, quadratic, and cubic cooling curve extrapolations

\begin{tabular}{c|l|c|c|c|c}
\hline \hline \multirow{2}{*}{$\begin{array}{c}\text { Run } \\
\text { length }\end{array}$} & \multirow{2}{*}{$\begin{array}{c}\text { Type of } \\
\text { run }\end{array}$} & $\begin{array}{c}\text { Calcu- } \\
\text { lated heat } \\
\text { loss cor- } \\
\text { rection }\end{array}$ & \multicolumn{3}{|c}{ Extrapolation error } \\
\cline { 4 - 6 } & & linear & quadratic & cubic \\
\hline $100 \mathrm{~s}$ & $E_{C}^{c}$ & $3.58 \%$ & $-0.36 \%$ & $0.03 \%$ & $0.04 \%$ \\
& $E_{C+j}^{c}$ & 0.12 & 0.24 & 0.01 & -0.03 \\
& $E_{C}^{r}$ & 0.00 & 0.00 & 0.00 & 0.00 \\
& & & & & $1.3 \%$ \\
& $E_{C}^{c}$ & $32.8 \%$ & $-12.7 \%$ & $-3.4 \%$ & -0.3 \\
& $E_{C+J}^{c}$ & 7.7 & 4.6 & 3.1 & -0.01 \\
& $E_{C}^{r}$ & 0.10 & 0.47 & -0.05 & -0.03 \\
\hline
\end{tabular}

time, and for the radiation case with a 1000-s exposure time.

Note particularly that even with quadratic or cubic extrapolation, calibration runs in the $\mathrm{C}+\mathrm{J}$ mode (which is equivalent to the quasi-adiabatic mode) cannot be extrapolated accurately if they are as long as $1000 \mathrm{~s}$. This is one of the reasons why calibration runs should be short, even when the radiation runs must be long. Another reason is to reduce the effect of a changing drift rate, which increases the uncertainty in longer runs.

An alternative method of operation would be to use a 2-body calorimeter and make radiation runs (mode $\mathrm{C}$ ) and calibration runs (mode $\mathrm{C}+\mathrm{J}$ ) of equal length. Then there would be no need to determine heat-loss corrections, thus avoiding errors associated with them. However, this alternative method is only reliable for constant-power radiation sources. In general, it pays to determine the heat-loss corrections, and to design the calorimeter so that they will always be small (i.e., with a thermally floating shield).

After a run, the calorimeter bodies are cooled by allowing air to leak into the vacuum system. In practice, it has been found that an increase in air pressure to a few tenths of a mm of mercury is sufficient to cool the bodies below their equilibrium temperatures. To restore equilibrium, the vacuum system is pumped out again, $R_{x}$ is returned to $R_{x}(0)$, and the calorimeter components are heated individually, in the following manner:

In mode $\mathrm{C}$, heat the core with the calibration heater until the bridge output is zero. Switch to mode J, and supply power to the jacket thermistor (by an auxiliary circuit not shown in fig. 13) until the bridge output is again zero. ${ }^{12}$ In mode $S$, heat the shield with the heater embedded in its back end (fig. 9), until the bridge output voltage is zero. The medium generally requires no restoration to equilibrium because of its thermoregulator.

Restoring the calorimeter components to their equilibrium temperatures is a process of successive approximations since the temperature of a given component will drift from its equilibrium value if the temperatures of adjacent components have not yet been equilibrated. This process can be shortened considerably, however, if the different components are heated simultaneously. Their temperatures must be observed with the circuit of figure 13 . The temperatures of core, jacket, shield and medium cannot be observed simultaneously, but with a little experience, the heat required for each component can be predicted. For short runs, such as those produced by the NBS linac, where a dose rate of $50 \mathrm{rads} / \mathrm{s}$ and an exposure time of $30 \mathrm{~s}$ were typical, the time for a complete cycle was of the order of $12 \mathrm{~min}$. For lower dose rates and longer runs, the cycling time is commensurately increased.

It is found on occasion that even after the initial temperatures have all been restored, the calorimetric

${ }^{12}$ The input to the bridge amplifier must be shorted while this is being done. In order to observe J during heating, it would be necessary to supply a separate heater for the jacket. 
bodies are slightly out of equilibrium which is presumably caused by changes in the ambient temperature. This can be observed by recording the temperature drift of the bodies on all the switch positions, and it can be corrected by selective heating or cooling of the bodies until the drifts are reduced to negligible values. The bridge balance can then be restored in the $\mathrm{C}, \mathrm{J}, \mathrm{S}$, and $\mathrm{M}$ modes by small adjustments of $R_{J}$, $R_{C}, R_{s}$, and $R_{m}$, and in the $\mathrm{C}+\mathrm{J}$ mode by making a small adjustment of the high-resistance shunt, $R_{4}$. This consists of a $600-\mathrm{k} \Omega$ fixed resistance in series with a $300-\mathrm{k} \Omega$ variable resistance which is initially set at mid-scale. This shunt reduces the sensitivity of the jacket thermistor by a small fraction of a percent, and since the calibration energy added to the jacket is a small fraction of the energy added to the core, it is only necessary to make a correction for this sensitivity change in very high-precision calibrations.

\section{Preliminary Tests}

The bench tests to be described in this section were made by heating the calorimeter electrically to check the mathematical description of section 2 , and to evaluate the systematic errors discussed in section 3 .

The calorimeter heat-transfer coefficients and heat capacities had to be evaluated before the bench tests could be analyzed. These parameters could be determined because the resistance of each thermistor was measured as a function of temperature. ${ }^{13}$ The temperature of any component could then be determined simply by measuring the resistance of its thermistor.

After the calorimeter had been assembled and evacuated to $10^{-5} \mathrm{~mm}$ of mercury, the heat-transfer coefficients $K_{1}$ and $K_{2}$ were determined by measuring $T_{1}(\infty), T_{2}(\infty)$, and $T_{3}(\infty)$ when an accurately measured electrical power, $P_{1}$, was dissipated in the core for a period of 16 hours. Equations (9) to (11) can be solved for $K_{1}$ and $K_{2}$, with $P_{2}=P_{3}=0$, to give:

and

$$
K_{1}=P_{1} /\left[T_{1}(\infty)-T_{2}(\infty)\right],
$$

$$
K_{2}=P_{1} /\left[T_{2}(\infty)-T_{3}(\infty)\right] .
$$

Equation (11) can also be solved for $K_{3}=P_{1} / T_{3}(\infty)$, but the small temperature rise was judged to lead to a very inaccurate measurement. Therefore, $K_{3}$ was computed, using an assumed emissivity of 0.1 for the aluminized mylar surfaces.

The heat capacities $C_{1}$ and $C_{2}$ were determined from 100 -s calibration runs in the $\mathrm{C}+\mathrm{J}$ mode, using eq (25). The recorder chart was calibrated by calculating the thermistor resistance changes from the change in the balancing resistor, $\Delta R_{x}$, needed to return to equilibrium, and then transforming this into a temperature change. The correction factor, $F_{C+J}^{C}$, was shown from calculations to be only 0.12 percent (fig. 6). The

${ }^{13}$ The thermistors selected for use in the core and jacket have sensitivities of -3.443 percent $/ \mathrm{K}$ and -3.438 percent $/ \mathrm{K}$ at $303 \mathrm{~K}$. Corrections must be made for this small difference in sensitivity in any high-accuracy measurements. shield heat capacity, $C_{3}$, was determined by comparing its mass to that of the core.

The final values for all of the calorimeter parameters are:

$$
\begin{aligned}
K_{1} & =0.785 \times 10^{-3} \pm 0.5 \%(1) \mathrm{W} / \mathrm{K}, \\
K_{2} & =1.109 \times 10^{-3} \pm 1.3 \%(6) \mathrm{W} / \mathrm{K}, \\
K_{3} & =5.2 \times 10^{-3} \pm 100 \%(1) \mathrm{W} / \mathrm{K}, \\
C_{1} & =1.081 \pm 0.4 \%(31) \mathrm{J} / \mathrm{K}, \\
C_{1} / C_{2} & =1.001 \pm 0.1 \%(1),
\end{aligned}
$$

and

$$
C_{3}=92 \pm 2 \%(1) \mathrm{J} / \mathrm{K},
$$

where the quoted uncertainties are standard errors of the mean and the numbers in parentheses are the degrees of freedom.

The first bench test consisted of a comparison of experimental temperature-time curves with calculated curves like those of figure 2 , using these values of the parameters. The results for measurements in the $\mathrm{C}$ and $\mathrm{C}+\mathrm{J}$ modes are shown in figure 15 for times up to $700 \mathrm{~s}$. The measured and calculated curves were normalized at the extrapolated peak of the $C+J$ curve. The RMS deviation between measured and calculated curves is of the order of 0.2 percent for times greater than $100 \mathrm{~s}$, which shows that the heat-loss corrections can be calculated accurately. The differences are larger when the heater is on, where the curves are much steeper. The experimental curve does not start to rise until about 2 seconds after power has been turned on, and continues to rise for about 2 seconds after it has been turned off. This is presumably a measure of the time required for heat to traverse the core from the heater to the temperature sensor.

The second bench test consisted of a comparison of electrical calibrations performed with the singlethermistor and two-thermistor bridges, as suggested in the penultimate paragraph of section 3 . The test was performed with an electrical power of $1400 \mu \mathrm{W}$ applied to the core for $100 \mathrm{~s}$. The time interval was accurately measured in terms of the NBS $100 \mathrm{~Hz}$ standard frequency. The test was performed over a period of four days, and consisted of 65 measurements of the fractional change in $R_{x}$ (see figs. 4 and 5) required to rebalance the bridge after the application of this power in either the $\mathrm{C}$ mode or its alternate, the $\mathrm{C}+\mathrm{J}$ mode. Each calorimeter component was restored to its initial equilibrium condition after each run. The averaged results are:

$$
\begin{aligned}
\frac{E_{C}^{c}}{E_{C+J}^{c}}= & \frac{T_{1}(\tau)\left(1+F_{C}^{c}\right)}{\left[T_{1}(\tau)+T_{2}(\tau)\right]\left(1+F_{C+J}^{c}\right)} \\
& =\frac{(0.0043332 \pm 0.025 \%)(1.0358 \pm 0.02 \%)}{(0.0044810 \pm 0.01 \%)(1.0012 \pm 0.002 \%)} \\
= & 1.0004 \pm 0.03 \%
\end{aligned}
$$




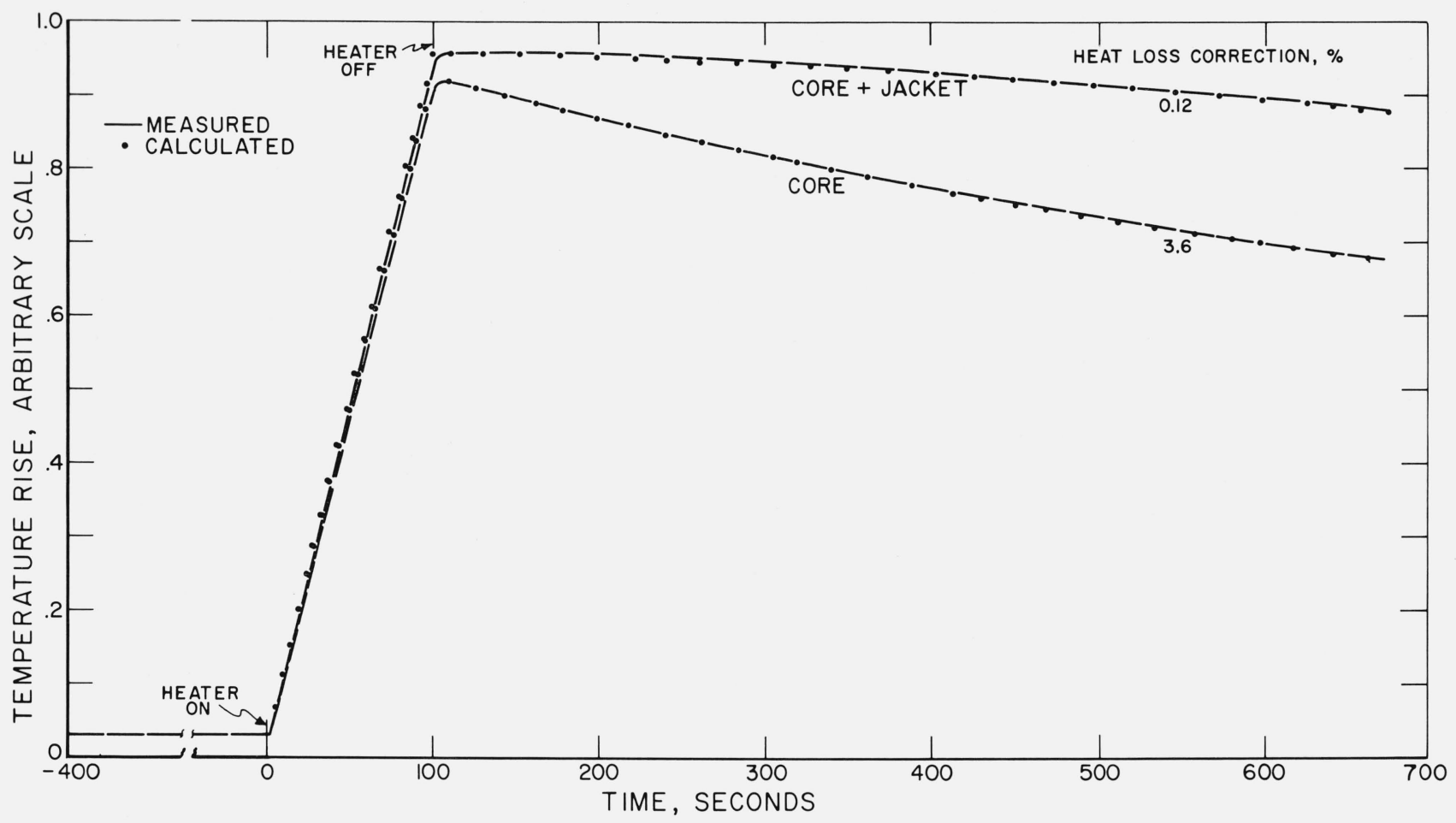

FIGURE 15. Comparison of measured (dashed curves) and calculated (dotted curves) run records that shows both the validity of the heat-loss calculations and the effectiveness of the jacket in reducing heat loss during electrical calibration.

The calculations were normalized at the extrapolated peak of the measured $\mathrm{C}+\mathrm{J}$ curve.

The observed temperature rises are given as fractional resistance changes, and the correction factors were calculated from eqs (24) and (26), using the temperature rises predicted by (4). The listed uncertainties are standard errors of the mean, and the number of degrees of freedom are 30 for each of the temperature rises, 2 for the numerator heat loss correction, and 6 for the denominator correction. The effective number of degrees of freedom in the final result was calculated to be 13 .

The third bench test was the consistency check described in the last paragraph of section 3. For operation as a quasi-adiabatic 2-body calorimeter, electrical power of $440 \mu \mathrm{W}$ was dissipated in both the core heater and the jacket thermistor (in excess of the $38 \mu \mathrm{W}$ bridge power) for $100 \mathrm{~s}$. The resistance change needed to rebalance the bridge in the singlethermistor, mode, $25 \mathrm{~s}$ after power was turned off, was recorded and measured. Measurements in this mode were compared with calibration runs in the twothermistor mode, where $440 \mu \mathrm{W}$ was supplied to the core alone. A total of 20 runs were made, alternating the two modes. The results are:

$\frac{T_{1}^{q}(\bar{t})}{T_{1}^{c}(\bar{t})+T_{2}^{c}(\bar{t})}=\frac{0.0013889 \pm 0.02 \%}{0.0013889 \pm 0.02 \%}$

$$
=1.0000 \pm 0.03 \%,
$$

where $\bar{t}=125 \mathrm{~s}$. The temperature rises given here are again inferred from fractional resistance changes (uncorrected for heat loss), and the uncertainties are again standard errors of the mean. ${ }^{14}$ Each of the temperature rises has 9 degrees of freedom, and the final result has 18.

\section{Radiation measurements}

The two calorimeters have been used with three sources of radiation, the NBS linear accelerator, the NBS cobalt-60 source, and the two-mile electron accelerator at the University of Stanford Linear Accelerator Center (SLAC), Stanford, California. The work will be reported only very briefly here, to illustrate the behavior of the two instruments in those situations.

The calorimeter used with the NBS linear accelerator has produced measurements of absorbed dose in graphite at depths up to $51 \mathrm{~g} / \mathrm{cm}^{2}$, using electron beams with energies from 15 to $50 \mathrm{MeV}$. These were compared with measurements of the specific ionization in air at the same depths. The results ${ }^{15}$ are in good agreement with experimental and theoretical results of other investigators.

The portable calorimeter was first tested in a cobalt60 beam that produced a dose rate near $10 \mathrm{rad} / \mathrm{min}$

${ }^{14}$ This electrical test would apply even for the case where $C_{1} \neq C_{2}$, and for an $n$-body calorimeter $(n>1)$ with different heat capacities and heat transfer coefficients. All that is necessary is to apply the powers for the same duration and in proportion to the sensitivities of the embedded sensors [1-3].

${ }_{15} \mathrm{~A}$ report is in manuscript at the time of writing (January, 1974). 
(fig. 16) in the calorimeter core. The temperature of the laboratory room was steady to within $0.3 \mathrm{~K}$. The standard deviation of the mean of eleven dose measurements under these conditions was less than one percent with exposure times between 15 to $24 \mathrm{~min}$.
The second test proves the equality of one-body and two-body calibrations, well within a tenth of a percent. That is, regardless of the temperature distribution in the core while it was being heated, it had an average surface temperature which increased steadily with

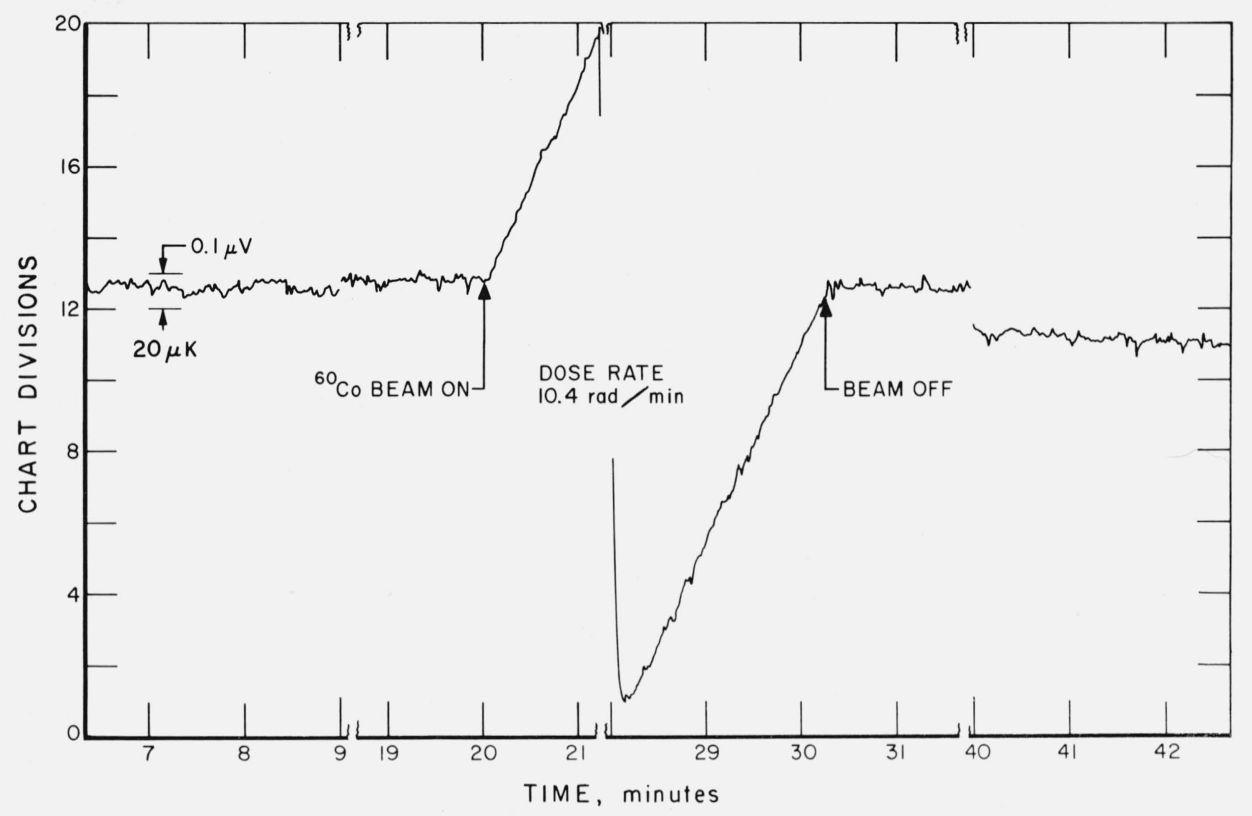

FIGURE 16. Response of the portable calorimeter to a dose rate of $10.4 \mathrm{rad} / \mathrm{min}$ produced by cobalt-60 radiation.

Note the breaks in the time scale.

The experimental conditions at SLAC were much more severe. The dose rates and exposure times were about the same as for the cobalt-60 measurements, but the SLAC beam was narrower than the core. The core, jacket, and shield received relative average dose rates of 10,6 , and 4 , respectively, resulting in heat-loss corrections ranging from 7 to 17 percent. In addition, the room temperature changed by $10 \mathrm{~K}$ during the day. Under these conditions, measurements by the portable calorimeter of the absorbed dose per incident $19.5-\mathrm{GeV}$ electron had a standard deviation of the mean of seven determinations of 1.3 percent [10].

\section{Discussion}

The first bench test discussed in section 6 shows that the mathematical description of calorimeter operation developed in section 2 is adequate for both one-body and two-body calibrations, except for the time lag. Temperature rises lag behind the application of power by about $2 \mathrm{~s}$, but soon after calibration power is turned off, the experimental time-temperature curves follow the theoretical curves to within a few tenths of 1 percent. increasing time, and it lost essentially the same amount of heat which would have been lost by a core whose uniform temperature was equal to this average temperature at all times. This result shows that the effects of thermal gradients in the core during calibration are negligible for both one-body and two-body operation.

The third bench test proves the equality of quasiadiabatic and heat-loss-compensated calibrations. The temperature difference between core and jacket is much smaller in the former case, from which it follows that errors which are a function of this difference are negligible. This includes errors caused by the transfer of heat in both the thermistor leads and the core heater leads.

Taken together, these tests show that both the core and the jacket thermistors read average temperature correctly when the calibration power is turned off and the transients have disappeared. After this has happened, the core (or jacket) temperature is uniform, except for the small radial drop (or rise) discussed in the third paragraph of section 3 . If either thermistor could see this radial drop (or rise) in temperature, it would be difficult for both the second and third bench tests to show equality.

It is unlikely that large effects of the four thermal gradients discussed in section 3 could cancel each 
other in both the second and third bench tests. We therefore conclude that the effects of all four are small, less than 0.1 percent, considering the precision of the measurements.

It should be noted that the third bench test is not really a comparison between calibrations with and without thermal gradients, since the quasi-adiabatic calibration uses two essentially point sources of power rather than uniformly distributed sources. It is felt that the thermal gradients in these two calibrations differed enough so that the results of this test are a good indication that the effects of thermal gradients are negligible. Nevertheless, it is possible that some undetected systematic error is present, and that the uncertainty in the heat-loss-compensated calibration is somewhat larger than the cited 0.1 percent.

Finally, mention should be made of a different proposal for increasing the sensitivity of a heat-losscompensated calorimeter [11]. This proposal is to embed thermistors with positive temperature coefficients of resistance (PTC) along with the negative-coefficient thermistors (NTC). Each PTC thermistor would be connected in a Wheatstone bridge arm adjacent to the one containing the NTC thermistor. Some PTC thermistors appear to have a useful sensitivity about twice that of the NTC thermistors, so it may be possible to triple the bridge output voltage per unit temperature rise without an accompanying noise increase.

\section{Summary}

A calorimeter for measuring the absorbed dose produced in graphite by ionizing radiation is described. Its design is based on compensation for heat lost from the calorimeter core during calibration by automatically adding the temperature of the surrounding jacket to the temperature of the core. The firstorder calorimeter theory is developed in detail, and four potential sources of systematic error are described. The construction of the calorimeter and the measurement and control techniques which have proven most effective are described in detail. Finally, test data are presented which show that the four systematic errors mentioned above are negligible for this calorimeter as it was used.

The principal author is indebted to his wife, Mary, whose patience contributed significantly to the achievements of this effort. We are also indebted to and take pleasure in thanking Ben Petree for his encouragement and understanding during this entire work. His many valuable discussions and contributions are appreciated. Thanks are also due to John S. Pruitt for his help in the preparation of the manuscript. This work was supported primarily by the National Cancer Institute, National Institutes of Health, Bethesda, Maryland. Some initial work was supported by the Division of Biology and Medicine of the United States Atomic Energy Commission, Washington, D.C.

\section{References}

[1] Domen, S. R., Heat-loss-compensated calorimeter, Nature 222, No. 5198, 1061 (June 1969).

[2] Domen, S. R., A heat-loss-compensated calorimeter and related theorems, J. Res. Nat. Bur. Stand. (U.S.), 73C (Eng. and Instr.), Nos. 1 and 2, 17-20 (Jan.-June 1969).

[3] Domen, S. R., U.S. Patent 3,665,762 (May 1972).

[4] White, W. P., The modern calorimeter, The Chem. Cat. Co., Inc., New York, 17 (1928).

[5] Persoz, B., Nouvelles methodes de mesure de la chaleur specifique vraie des metaux a haute temperature, Ann. Phys. (PARIS) 14, 237-301 (July-Dec. 1940).

[6] Ginnings, D. C., and West, E. D., in Experimental Thermodynamics, Ed. J. P. McCullough, and D. W. Scott, Vol. 1, 125-127 (Plenum Press, New York, N.Y., 1968).

[7] Laughlin, J. S., and Genna, S., In Radiation Dosimetry, Ed. F. H. Attix, and W. C. Roesch, Vol. 11, 411-415 (Academic Press, Inc., New York, N.Y., 1966).

[8] Petree, B., and Ward, G., The construction of calorimeters for the measurement of absorbed dose, Nat. Bur. Stand. (U.S.), Tech. Note 163,36 pages (Nov. 1962).

[9] Engelke, B. A., and Hohlfeld, K., Einflub von temperaturgradienten im absorber bei der kalorimetrischen bestimmung der energiedosis PTB Mitt. 81, No. 3, 185-189 (June 1971).

[10] Kase, K. R., and Domen, S. R., Calorimetric and ionization measurements of stopping power in carbon for $19.5 \mathrm{GeV}$ electrons, Nucl. Instr. Meths., 118, No. 2, 469-475 (1974).

[11] Domen, S. R., Petree, B., and Lamperti, P. J., The heat-losscompensated calorimeter for measurement of absorbed dose, Twelfth Annual Meeting of the AAPM, Wash., D.C. Quart. Bull. 4, No. 2, 71 (June 1970).

(Paper 78A5-834) 